Agro-Science Journal of Tropical Agriculture, Food, Environment and Extension

Volume 11 Number 1 Januarv 2012 nn $55-63$

ISSN 1119-7455

\title{
ESTIMATION OF HETEROSIS AND INBREEDING DEPRESSION IN QUANTITATIVE TRAITS OF RICE \\ (Oryza sativa L.)
}

\author{
Onyia $^{1}$, V.N., Obi, ${ }^{1}$ I.U and Anyanwu ${ }^{2}$ C. P \\ ${ }^{1}$ Department of Crop Science, University of Nigeria Nsukka \\ ${ }^{2}$ Department of Crop Science and Technology, F.U.T.O
}

\begin{abstract}
It is important to know the degree and direction of heterosis for its commercial exploitation. Heterosis and in-breeding depression were estimated in $8 x 8$ half diallel crosses of rice. The planted materials consisted ofeight parental inbred lines, their $F_{1}$ hybrids and $F_{2}$ populations using randomized complete block design with three replications. Data were collected on number of days to $50 \%$ flowering, plant height, number of tillers/plant, number of panicles/hill, panicle length, number of spikelets/panicle, number of fertile spikelets/panicle, number of days to maturity, 1000-seed weight and grain yield. Significant genetic differences were observed among the parents, their $F_{1}$ hybrids and $F_{2}$ populations for all the characters under study. Panicle length and number of

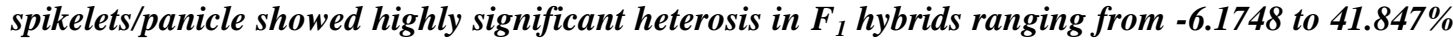
and -8.6957 to $41.847 \%$, respectively while in breeding depression in the $F_{2}$ population ranged from -3.93 to $13.2231 \%$ and 3.6364 to $25.85 \%$ respectively. $F_{1}$ hybrids showed low level of heterosis in number of days to flowering (-11.25 to 11.95\%),.The parent WAB 450-1-B-163-41 proved itself to be a good general combiner by making higher contribution towards heterosis both in $F_{1}$ hybrids and in $F_{2}$ population
\end{abstract}

Keywords: Genetic basis, Oryza sativa, hybrid vigor, quantitative traits, inbreeding depression

\section{INTRODUCTION}

Rice (Oryza sativa L.) is a well known cereal crop grown in almost every part of the world. Although, the plant is naturally self pollinated, strong heterosis is observed in their $\mathrm{F}_{1}$ hybrids. The term heterosis was coined by Shull (1908) for quantitative measure of superiority of $F_{1}$ over its parents. The phenomenon of heterosis has been a powerful force in the evolution of plants and has been exploited extensively in crop production (Birchler et al., 2003). Heterosis in rice can often be poorly expressed as reported by some scientists (Mohammed and Mohanty 1992; Ram 1992, Virekananden and Giridheram 1995).

The phenomenon of heterosis has been observed in many self-pollinated crop species including several of the grain legumes. It is commonly found that the level of heterosis exhibited by a hybrid is a function of the genetic divergence between parents. Heterosis may be positive or negative. Depending on breeding objectives both positive and negative heterosis can be useful for crop improvement. Heterosis is a highly cross specific phenomenon. To successfully use heterosis in grain yield improvement, parental genotypes need to have a high yield potential. The exploration of hybrid vigor is widely recognized as the only readily available means to raise the genetic yield ceiling in areas where yields have already approached their potential. In this approach, developing highly heterotic rice hybrids with superior yield performance and evaluating them across environments are important. (Sitaramaiah et al., 1998). The project was initiated with the objectives to determine the heterotic effects in $\mathrm{F}_{1}$ hybrids and the inbreeding of the plant behavior in both hybrid and selfed conditions.

\section{MATERIALS AND METHODS}

The research was conducted at the experimental field of the National Cereals Research Institute (NCRI), Amakanma sub- 
station Umuahia, Abia State. Eight upland rice breeding lines: IRAT 317, WAB 35-1-FX, IR 47-701-6-3-1, IRAT 239, WAB 450-1-163-14, WAB35-2-FX, WAB 56-144 FX and WAB 56-100) were crossed in a full diallel pattern.

In the production of $\mathrm{F}_{1}$ 'sfour crossing blocks were established at two weekly intervals consisting of eight plots per block. Each crossing block measured $12.5 \mathrm{~m} \mathrm{x} 1 \mathrm{~m}$ and there plot in the crossing block measured $12.5 \mathrm{~m} \mathrm{x} 1 \mathrm{~m}$ and there plot in the crossing block measured $1 \mathrm{~m} \mathrm{x} 1 \mathrm{~m}$ and a $0.5 \mathrm{~m}$ alley separated each plot. Rice seeds from each of the selected eight breeding lines were seeded in each plot at a spacing of $20 \mathrm{~cm} \mathrm{x}$ $20 \mathrm{~cm}$. The purpose of establishing the crossing blocks at two weeks interval was to synchronize the flowering time of the breeding lines for the purpose of crossing. A total of four plantings were done. The $\mathrm{F}_{2}$ generation was obtained by allowing natural self-pollination of the back crosses. The hybrids and parents were evaluated in a randomized complete block design at a spacing of $20 \mathrm{~cm} \times 20 \mathrm{~cm}$ in lines. Each genotype was grown in a single row of ten plants. Data were collected from all plants, leaving one border plant on each side of each genotype (Dhaliwal and Sharma, 1990). Observations were recorded on 12 characters namely: number of days to $50 \%$ flowering, plant height, number of tillers/plants, number of tillers $/ \mathrm{m}^{2}$, number of panicles/hill, number of panicles $/ \mathrm{m}^{2}$, panicle length, number of spikelets/panicle, spikelet fertility, number of days to maturity, 1000-grain weight and grain yield.

Heterosis and inbreeding depression were estimated according to Falconer and MacKay (1996) for $F_{1}$ hybrids and inbreeding depression for $F_{2}$ populations were estimated using the following formulae:

Mid parent Heterosis $(\%)=\frac{\overline{F_{1}}-M P}{M P} \times \frac{100}{1}$

Where

$\mathrm{F}_{1} \quad=$ Mean of $\mathrm{F}_{1}$ hybrid for a trait

MP $=$ Mid parent

Inbreeding depression on $\mathrm{F}_{2}$ from $\mathrm{F}_{1}(\%)=$ $\frac{\left(F_{1}-\bar{F}_{2}\right)}{\overline{F_{1}}}$

$\begin{aligned} & \frac{\text { Where }}{\mathrm{F}_{2}} \\ & \text { trait }\end{aligned}=\quad$ Mean of $\mathrm{F}_{2}$ population for $\mathrm{a}$

The analysis of variance was performed according to the procedure outlined by Steel and Torrie (1980) and separation of treatment means for significant effect was done using the least significant difference (LSD) according to Obi (2002).

\section{RESULTS AND DISCUSSION}

The analysis of variance table presented in Table 1 show significant effect $(\mathrm{P}=0.05)$ for the parameters studied except for number of tillers/plant, number of tillers $/ \mathrm{m}^{2}$ and number of panicle/hill which were non significant. In Table 2 is presented the mean performance of the twelve agronomic attributes of the eight size breeding lines used for the study. The results of the different morphological characters of the eight rice breeding lines, their twenty-eight hybrids and $\mathrm{F}_{2}$ populations are presented in Table 3.

In the present study, different cross combinations were tested in order to develop high yielding hybrid rice varieties and some were found to be promising. The stability of hybrids was checked through their performance in the $F_{2}$ generation, and variable inbreeding depression was also observed for the studied traits in the different crosses.

Development of high yielding early maturing varieties is a highly desirable quality in most rice breeding programs. Among the twenty eight crosses, highly negative heterosis was observed in some crosses. While some of the crosses such as WAB 56-144-FX X WAB 56-100 and IRAT $317 \times$ WAB 56-100 showed positive heterosis, others such as WAB 450-1-B-163-41 X WAB 35-2-FX and WAB 35-2-FX X IRAT 239 showed negative heterosis for both days to $50 \%$ flowering and number of days to maturity which suggests the possibility of developing early maturity lines from these combination. Negative heterosis for earliness was also reported by Khaleque et al., (1977) and Nuruzzaman et al., (2002) in rice.

The number of panicles is one of the components used in determining grain yield. There were increase in panicle number in these hybrids WAB 450-1-B-163-41 x IRAT 317, IRAT 317 x WAB 56-144-FX, WAB 35-1-FX x WAB 56-144-FX, IR 47-701-6-3-1 x WAB 352-FX, RAT 317 x IR 47-701-6-3-1, IRAT 317 x IRAT 239 and IR 47-701-6-3-1 x IRAT-239 which had 8 panicles/hill each and WAB 35-1FXx WAB 35-2-FX that had 9 panicles/hill over their respective parents. Increase in panicle number was earlier observed by Singh et al. (1980), Anandakumah and Sree Rangasamy (1986). The results show that two crosses IRAT 317 x IRAT 239 and IR 47-701-6-3-1 x IRAT 239 showed highly significant positive heterosis values of $45 \%$ in panicle number per hill., respectively. 
Table 1: Form of Analysis of variance showing sources of Variation, Degrees of Freedom and Mean Square Estimates of Eight Rice breeding lines for twelve (12) Plant Attributes.

\begin{tabular}{|c|c|c|c|c|c|c|c|c|c|c|c|c|c|}
\hline SV & DF & $50 \%$ & Plant ht & Tiller /plant & Tillers/M ${ }^{2}$ & Panicles /hill & $\begin{array}{l}\text { Panicles } \\
/ \mathrm{M}^{2}\end{array}$ & Panicle ength & Spikelets panicles & FS/P & DTM & GW & GY \\
\hline Block & 3 & 2.083 & 2.57 & 5.583 & 6.948 & 10.417 & $\begin{array}{l}54.88 \\
\end{array}$ & 6.38 & 631.2 & 347.7 & 2.088 & 1.616 & 0.2545 \\
\hline Genotypes & 7 & $214.2^{* *}$ & $936^{* *}$ & $8.500 \mathrm{~ns}$ & $5620 \mathrm{~ns}$ & $4.286 \mathrm{~ns}$ & $3346^{*}$ & $27.86^{*}$ & $14171^{* *}$ & $\begin{array}{l}7673^{*} \\
*\end{array}$ & $214.2^{* *}$ & $153.3^{* * *}$ & 5.096 \\
\hline $\begin{array}{l}\text { Error } \\
\text { Total }\end{array}$ & $\begin{array}{l}21 \\
31\end{array}$ & 2.179 & 17.99 & 4.345 & 0.970 & 3.464 & 1001 & 10.79 & 364.70 & 368.0 & 2.179 & 1.209 & 0.0823 \\
\hline
\end{tabular}

Table 2. Mean Performance of the ten agronomic attributes of the eight rice breeding lines evaluated at the NCRI Umudike, Abia State.

\begin{tabular}{|c|c|c|c|c|c|c|c|c|c|c|c|c|}
\hline $\begin{array}{l}\text { Genotypes } \\
\text { Parents }\end{array}$ & $\begin{array}{l}\text { Days to } 50 \% \\
\text { Flowering }\end{array}$ & $\begin{array}{l}\text { Plant } \\
\text { height }\end{array}$ & $\begin{array}{l}\text { No of } \\
\text { Tillers/ } \\
\text { Plant }\end{array}$ & $\begin{array}{l}\text { No of } \\
\text { Tillers/M } \mathbf{M}^{2}\end{array}$ & $\begin{array}{l}\text { No of } \\
\text { Panicles/Hill }\end{array}$ & $\begin{array}{l}\text { No of } \\
\text { Panicles/ } \\
\mathrm{M}^{2}\end{array}$ & $\begin{array}{l}\text { Panicle } \\
\text { Length }\end{array}$ & $\begin{array}{l}\text { No of } \\
\text { Spikelet/ } \\
\text { Panicle }\end{array}$ & $\begin{array}{l}\text { No of } \\
\text { Fertile } \\
\text { Spikelets } \\
\text { /Panicle }\end{array}$ & $\begin{array}{l}\text { No of } \\
\text { Days to } \\
\text { Maturity }\end{array}$ & $\begin{array}{l}1000 \\
\text { Grain } \\
\text { Weight }\end{array}$ & $\begin{array}{l}\text { Grain } \\
\text { Yield }\end{array}$ \\
\hline Grand mean & 78.87 & 92.25 & 10.88 & 275.4 & 6.25 & 164.50 & 24.58 & 128.40 & 106.60 & 108.87 & 30.58 & 3.681 \\
\hline WAB 450-1-B-163-41 & 71 & 103.22 & 11 & 283 & 6 & 145 & 24.53 & 272 & 211 & 101 & 34.43 & 5.87 \\
\hline IRAT 317 & 90 & 86.70 & 12 & 302 & 7 & 195 & 21.98 & 96 & 88 & 120 & 23.10 & 2.810 \\
\hline WAB35-1-FX & 84 & 66 & 12 & 310 & 7 & 165 & 23.52 & 116 & 91 & 114 & 39.33 & 3.850 \\
\hline IR 47-701-6-3-1 & 85 & 93.6 & 11 & 282 & 7 & 178 & 20.57 & 80 & 71 & 115 & 22.87 & 2.310 \\
\hline WAB35-2-FX & 75 & 77.44 & 13 & 322 & 7 & 205 & 24.00 & 126 & 82 & 105 & 35.63 & 4.55 \\
\hline WAB56-144-FX & 76 & 114.22 & 10 & 255 & 6 & 148 & 27.75 & 111 & 102 & 106 & 33.26 & 3.570 \\
\hline IRAT 239 & 81 & 101.5 & 9 & 227 & 4 & 115 & 26.32 & 123 & 108 & 111 & 25.23 & 2.850 \\
\hline WAB 56-100 & 69 & 95.33 & 9 & 222 & 6 & 165 & 27.98 & 111 & 100 & 99 & 30.82 & 3.640 \\
\hline F-LSD $(\mathrm{P}=0.05)$ & 2.170 & 6.237 & 3.065 & 80.14 & 2.737 & 46.52 & 4.830 & 28.08 & 28.21 & 2.170 & 1.67 & 0.4217 \\
\hline
\end{tabular}


Estimation of Heterosis and Inbreeding Depression in Rice

Table 3. Mean values of the agronomic characters of the parents, $F_{1}$ 's, $F_{2}$ 's , mid parent heterosis ( $\%$ het) for $F_{1}$ hybrids and Inbreeding depression (\% ID for $F_{2}$ populations) in the rice breeding lines.

\begin{tabular}{|c|c|c|c|c|c|c|c|c|c|c|c|c|c|c|}
\hline & & & Number of & ays to & $\%$ flowe & & Plant & eight (cm) & & ber of till & olant & & & \\
\hline Cro & $\mathrm{s}$ & Ses & $F_{1}$ & $\mathrm{~F}_{2}$ & $\%$ Het & $\%$ ID & $\mathrm{F}_{1}$ & $\mathrm{~F}_{2}$ & $\%$ Het & $\% \mathrm{ID}$ & $\mathrm{F}_{1}$ & $\mathrm{~F}_{2}$ & $\% \mathrm{Het}$ & $\% \mathrm{ID}$ \\
\hline WAB 450-1-B-163-41 & $\mathrm{X}$ & WAB35-2-FX & 68 & 68 & -6.8493 & 0 & 92.55 & 87.32 & 2.457 & 1.974 & 12 & 10 & 0 & 0 \\
\hline WAB 450-1-B-163-41 & $\mathrm{X}$ & WAB56-144-FX & 71 & 72 & -3.4014 & -1.4085 & 115.2 & 109.4 & 5.960 & 5.034 & 12 & 10 & 14.28 & 16.66 \\
\hline WAB 450-1-B-163-41 & $\mathrm{X}$ & WAB 56-100 & 70 & 71 & -1.4085 & 0 & 101. & 98.48 & 0.950 & 5.651 & 10 & 9 & -9.909 & 16.66 \\
\hline WAB35-2-FX & $\mathrm{X}$ & WAB56-144-FX & 74 & 74 & -1.9868 & 0 & 81.75 & 77.44 & -14.6 & 5.272 & 13 & 12 & 13.04 & 7.693 \\
\hline WAB35-2-FX & $\mathrm{X}$ & WAB 56-100 & 73 & 72 & 1.3889 & 1.3699 & 81.03 & 87.80 & -6.204 & -1.835 & 12 & 11 & 9.091 & 8.333 \\
\hline WAB56-144-FX & $\mathrm{X}$ & WAB 56-100 & 75 & 73 & 3.4483 & 2.6667 & 110.7 & 102.7 & 5.678 & 7.206 & 12 & 11 & 26.31 & 8.3333 \\
\hline WAB 450-1-B-163-41 & $\mathrm{X}$ & IRAT 317 & 81 & 81 & 0.6211 & 0 & 98.70 & 96.50 & 3.938 & 2.290 & 12 & 11 & 4.347 & 8.333 \\
\hline WAB 450-1-B-163-41 & $\mathrm{X}$ & WAB35-1-FX & 74 & 75 & -4.516 & -1.3514 & 88.67 & 78.07 & 4.7985 & 11.954 & 14 & 12 & 21.73 & 14.25 \\
\hline WAB 450-1-B-163-41 & $\mathrm{X}$ & IR 47-701-6-3-1 & 78 & 78 & 0 & -1.3514 & 99.80 & 97.50 & 1.4125 & 11.954 & 13 & 11 & 18.18 & 14.25 \\
\hline WAB 450-1-B-163-41 & $\mathrm{X}$ & IRAT 239 & 78 & 77 & 2.6316 & 0 & 108.93 & 97.25 & 6.4185 & 2.3046 & 11 & 11 & 10 & 15.38 \\
\hline WAB35-2-FX & $\mathrm{X}$ & IRAT 239 & 71 & 73 & -8.874 & 1.2821 & 79.90 & 96.90 & -10.696 & 10.722 & 12 & 12 & 9.090 & 0 \\
\hline WAB56-144-FX & $\mathrm{X}$ & IRAT 239 & 71 & 74 & -9.534 & -2.8169 & 116.22 & 109.30 & 15.3548 & -21.26 & 12 & 10 & 26.31 & 0 \\
\hline IRAT 317 & $\mathrm{X}$ & WAB35-2-FX & 85 & 83 & 3.0303 & 2.3529 & 80.15 & 81.40 & -2.339 & -1.559 & 11 & 10 & -6.6667 & 25 \\
\hline IRAT 317 & $\mathrm{X}$ & WAB56-144-FX & 86 & 84 & 3.6145 & 2.3256 & 93.80 & 98.75 & -6.629 & -5.277 & 12 & 11 & 23.6769 & 4.348 \\
\hline IRAT 317 & $\mathrm{X}$ & WAB 56-100 & 89 & 84 & 11.9500 & 5.6180 & 90.25 & 90.38 & -0.846 & -0.144 & 12 & 11 & 7.6923 & 4.7619 \\
\hline WAB35-1-FX & $\mathrm{X}$ & WAB35-2-FX & 81 & 80 & 1.8868 & 1.2346 & 69.71 & 69.40 & -4.196 & -1.004 & 15 & 12 & 20 & 13.0443 \\
\hline WAB35-1-FX & $\mathrm{X}$ & WAB56-144-FX & 71 & 73 & -11.25 & -2.8169 & 82.40 & 78.10 & -8.556 & 5.2184 & 10 & 9 & 23.0769 & 14.2857 \\
\hline WAB35-1-FX & $\mathrm{X}$ & WAB 56-100 & 75 & 78 & -1.9608 & -4 & 73.44 & 71.40 & $-8,962$ & 2.7778 & 12 & 11 & 7.6923 & 10 \\
\hline IR 47-701-6-3-1 & $\mathrm{X}$ & WAB35-2-FX & 80 & 80 & 0 & 0 & 80.55 & 82.80 & -5.811 & -2.793 & 14 & 10 & 6.6667 & 25 \\
\hline IR 47-701-6-3-1 & $\mathrm{X}$ & WAB56-144-FX & 82 & 81 & 1.8634 & 1.2195 & 105.60 & 103.95 & 1.6266 & 1.5625 & 12 & 13 & 1.3575 & 4.348 \\
\hline IR 47-701-6-3-1 & $\mathrm{X}$ & IRAT 239 & 80 & 78 & 3.8961 & 2.500 & 94.00 & 94.00 & -0.486 & 0 & 13 & 10 & 13.4021 & 4.7619 \\
\hline IRAT 239 & $\mathrm{X}$ & WAB 56-100 & 80 & 77 & 6.6667 & 3.75 & 96.50 & 97.31 & -0.944 & -0.839 & 11 & 10 & 4.7619 & 13.0443 \\
\hline IRAT 317 & $\mathrm{X}$ & WAB35-1-FX & 90 & 83 & 3.4483 & 2.222 & 75.00 & 75.00 & 2.2495 & -1.0484 & 15 & 14 & 25 & 8.333 \\
\hline IRAT 317 & $\mathrm{X}$ & IR 47-701-6-3-1 & 90 & 89 & 2.8571 & 2.222 & 89.66 & 89.66 & 0.5435 & 2.667 & 12 & 11 & 4.348 & 6.6667 \\
\hline IRAT 317 & $\mathrm{X}$ & IRAT 239 & 88 & 86 & 2.9240 & .272 & 94.00 & 94.00 & -10.106 & 1.1702 & 11 & 10 & 4.7619 & 9.0909 \\
\hline WAB35-1-FX & $\mathrm{X}$ & IR 47-701-6-3-1 & 80 & 82 & -5.3254 & 2.500 & 70.43 & 70.43 & -117419 & -0.8093 & 13 & 12 & 13.0443 & 7.6923 \\
\hline WAB35-1-FX & $\mathrm{X}$ & IRAT 239 & 85 & 83 & 3.0303 & 2.3529 & 69.00 & 69.00 & 17.6119 & -2.7536 & 12 & 11 & 14.2857 & 8.333 \\
\hline IR 47-701-6-3-1 & $\mathrm{X}$ & IRAT 239 & 84 & 83 & 1.2048 & 1.1905 & 95.41 & 95.41 & -2.1957 & -4.0771 & 11 & 11 & 10 & 0 \\
\hline F-LSD $(\mathrm{p}=0.05)$ & & & 2.182 & 3.23 & - & - & 4.371 & 5.3721 & - & - & 2.359 & 2.011 & - & - \\
\hline
\end{tabular}


Onyia $^{1}$, V.N., Obi, ${ }^{1}$ I.U and Anyanwu ${ }^{2}$ C. P

Table 3 (cont). Mean values of the agronomic characters of the parents, $F_{1}$ 's, $F_{2}$ 's , mid parent heterosis ( $\%$ het) for $F_{1}$ hybrids and Inbreeding depression ( $\% \mathrm{ID}$ for $\mathrm{F}_{2}$ populations) in the rice breeding lines.

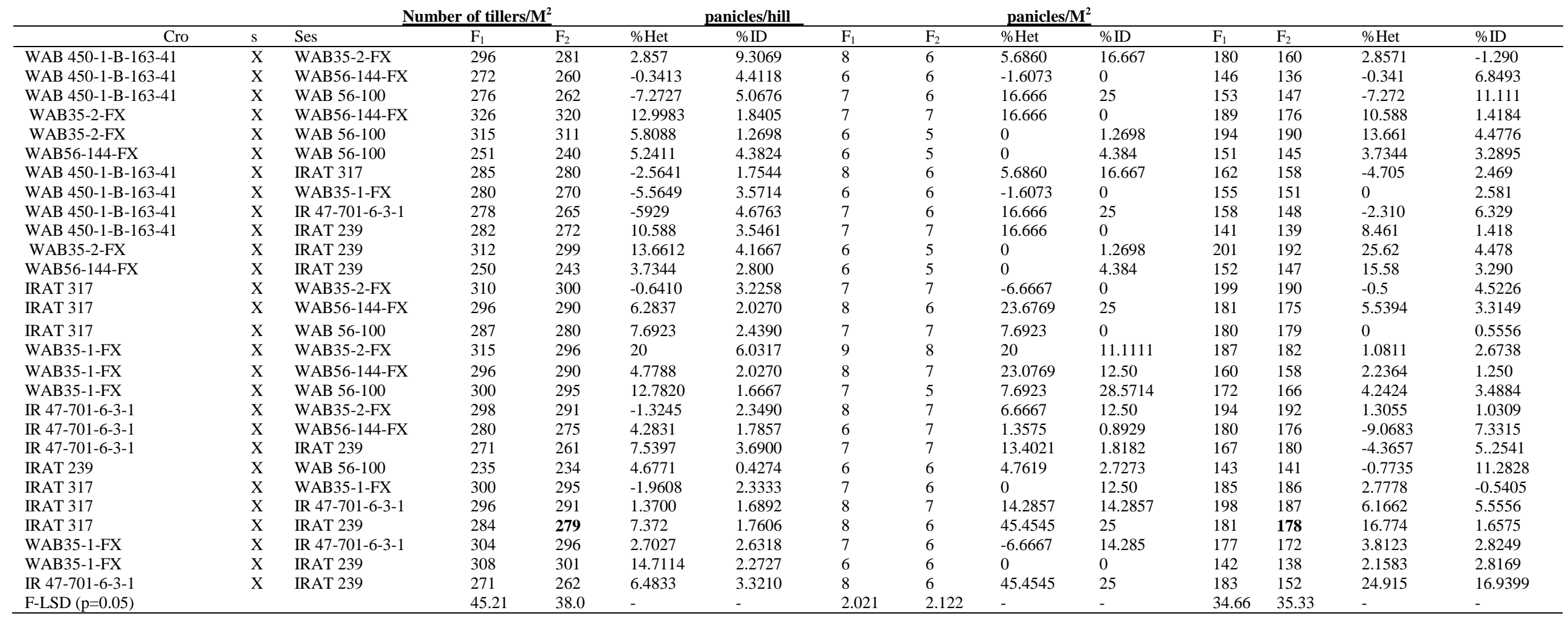


Estimation of Heterosis and Inbreeding Depression in Rice

Table 3 (cont). Mean values of the agronomic characters of the parents, $F_{1}$ 's, $F_{2}$ 's, mid parent heterosis ( $\%$ het) for $F_{1}$ hybrids and Inbreeding depression (\% ID for $\mathrm{F}_{2}$ populations) in the rice breeding lines.

\begin{tabular}{|c|c|c|c|c|c|c|c|c|c|c|c|c|c|c|}
\hline \multirow[b]{2}{*}{ Cro } & \multirow[b]{2}{*}{$\mathrm{s}$} & \multirow[b]{2}{*}{ Ses } & & \multicolumn{2}{|c|}{ Panicle length $(\mathrm{cm})$} & \multicolumn{3}{|c|}{ Number of spikelets/panicle } & \multicolumn{4}{|c|}{$\underline{\text { Number of fertile spikelets/panicle }}$} & \multirow[b]{2}{*}{$\% \mathrm{Het}$} & \multirow[b]{2}{*}{$\% \mathrm{ID}$} \\
\hline & & & $F_{1}$ & $\mathrm{~F}_{2}$ & $\% \mathrm{Het}$ & $\%$ ID & $\mathrm{F}_{1}$ & $\mathrm{~F}_{2}$ & $\% \mathrm{Het}$ & $\%$ ID & $\mathrm{F}_{1}$ & $\mathrm{~F}_{2}$ & & \\
\hline WAB 450-1-B-163-41 & $\mathrm{X}$ & WAB35-2-FX & 25.65 & 24.62 & 5.686 & 12.14 & 213 & 205 & 7.0352 & 25.848 & 200 & 195 & 36.518 & 31.832 \\
\hline WAB 450-1-B-163-41 & $\mathrm{X}$ & WAB56-144-FX & 25.71 & 24.00 & -1.607 & 6.651 & 218 & 210 & 13.838 & 3.6697 & 184 & 164 & 17.577 & 10.869 \\
\hline WAB 450-1-B-163-41 & $\mathrm{X}$ & WAB 56-100 & 29.45 & 23.82 & 5.253 & 4.015 & 241 & 221 & -11.39 & 3.7559 & 205 & 195 & -2.843 & 2.500 \\
\hline WAB35-2-FX & $\mathrm{X}$ & WAB56-144-FX & 24.31 & 22.75 & -5.99 & 6.417 & 120 & 113 & 1.2658 & 5.8333 & 88 & 81 & -4.347 & 7.9545 \\
\hline WAB35-2-FX & $\mathrm{X}$ & WAB 56-100 & 24.87 & 24.80 & -4.30 & 0.281 & 118 & 112 & -0.4219 & 5.0847 & 90 & 87 & -1.098 & 3.3333 \\
\hline WAB56-144-FX & $\mathrm{X}$ & WAB 56-100 & 26.80 & 24.35 & -3.77 & 9.141 & 110 & 106 & -0.900 & 3.6364 & 100 & 90 & -0.990 & 10.00 \\
\hline WAB 450-1-B-163-41 & $\mathrm{X}$ & IRAT 317 & 23.51 & 22.51 & 41.847 & 4.253 & 261 & 187 & 41.847 & 4.2126 & 200 & 187 & 33.779 & 6.5 \\
\hline WAB 450-1-B-163-41 & $\mathrm{X}$ & WAB35-1-FX & 23.52 & 24.21 & 35.051 & -3.93 & 262 & 163 & 35.051 & 4.1984 & 190 & 163 & 25.827 & 14.210 \\
\hline WAB 450-1-B-163-41 & $\mathrm{X}$ & IR 47-701-6-3-1 & 23.67 & 23.21 & 27.840 & -3.93 & 225 & 172 & 27.840 & 4.1984 & 180 & 172 & 27.659 & 14.210 \\
\hline WAB 450-1-B-163-41 & $\mathrm{X}$ & IRAT 239 & 25.01 & 24.21 & 20.607 & 1.943 & 254 & 192 & 20.607 & 11.111 & 180 & 172 & 12.852 & 4.4444 \\
\hline WAB35-2-FX & $\mathrm{X}$ & IRAT 239 & 24.82 & 23.40 & 2.008 & 3.198 & 127 & 88 & 2.008 & 7.0866 & 97 & 88 & 2.1053 & -6.666 \\
\hline WAB56-144-FX & $\mathrm{X}$ & IRAT 239 & 27.11 & 26.22 & 4.2735 & 5.721 & 122 & 100 & 4.2735 & 13.385 & 106 & 100 & 0.9524 & 9.2784 \\
\hline IRAT 317 & $\mathrm{X}$ & WAB35-2-FX & 22.66 & 20.62 & -1.430 & 9.0026 & 120 & 113 & 8.1281 & 5.833 & 85 & 80 & 0 & 5.8824 \\
\hline IRAT 317 & $\mathrm{X}$ & WAB56-144-FX & 23.84 & 21.21 & -4.0644 & 11.0319 & 113 & 96 & 9.1787 & 15.0442 & 108 & 83 & 13.6842 & 23.1481 \\
\hline IRAT 317 & $\mathrm{X}$ & WAB 56-100 & 24.45 & 23.41 & -2.1217 & 4.2530 & 108 & 104 & 4.3478 & 4.6296 & 95 & 90 & 1.0638 & 5.2633 \\
\hline WAB35-1-FX & $\mathrm{X}$ & WAB35-2-FX & 21.66 & 19.49 & -8.838 & 10.0185 & 118 & 102 & -2.4793 & 13.5593 & 87 & 72 & 0.5780 & 17.2413 \\
\hline WAB35-1-FX & $\mathrm{X}$ & WAB56-144-FX & 24.21 & 22.20 & 5.5035 & 8.3024 & 116 & 101 & 2.2026 & 14.736 & 95 & 81 & -1.5544 & 14.736 \\
\hline WAB35-1-FX & $\mathrm{X}$ & WAB 56-100 & 34.16 & 23.01 & -6.1748 & 4.7599 & 114 & 100 & 0.4405 & 12.2807 & 84 & 74 & -12.041 & 11.9048 \\
\hline IR 47-701-6-3-1 & $\mathrm{X}$ & WAB35-2-FX & 21.27 & 20.11 & -4.5760 & 5.4537 & 110 & 101 & 6.791 & 8.1818 & 76 & 70 & -7.3171 & 7.8947 \\
\hline IR 47-701-6-3-1 & $\mathrm{X}$ & WAB56-144-FX & 21.96 & 20.35 & -2.6178 & 6.4516 & 93 & 88 & -8.6957 & 10.7143 & 84 & 75 & -3.5464 & 3.0616 \\
\hline IR 47-701-6-3-1 & $\mathrm{X}$ & IRAT 239 & 23.22 & 22.00 & -1.5707 & 9.5745 & 98 & 85 & -7.6923 & 4.7619 & 84 & 80 & -0.8939 & 3.7204 \\
\hline IRAT 239 & $\mathrm{X}$ & WAB 56-100 & 26.94 & 23.90 & 3.4188 & 13.2231 & 121 & 105 & 1.9231 & 10.3774 & 106 & 95 & -7.9201 & 2.3247 \\
\hline IRAT 317 & $\mathrm{X}$ & WAB35-1-FX & 22.02 & 20.63 & 12.2855 & 12.2855 & 108 & 98 & 1.8868 & 17.3469 & 90 & 85 & 0.5587 & 13.333 \\
\hline IRAT 317 & $\mathrm{X}$ & IR 47-701-6-3-1 & 22.14 & 19.42 & 6.3124 & 6.3124 & 98 & 81 & 0.1136 & 9.2593 & 90 & 78 & 13.2075 & 5.5556 \\
\hline IRAT 317 & $\mathrm{X}$ & IRAT 239 & 22.04 & 20.0 & 9.0290 & 9.0290 & 108 & 101 & -1.3699 & 6.4815 & 91 & 87 & -7.1429 & 4.3956 \\
\hline WAB35-1-FX & $\mathrm{X}$ & IR 47-701-6-3-1 & 30.14 & 28.32 & 6.0385 & 6.0385 & 114 & 100 & 10.6796 & 12.2807 & 89 & 76 & 9.8765 & 14.6.67 \\
\hline WAB35-1-FX & $\mathrm{X}$ & IRAT 239 & 23.87 & 22.63 & 5.1948 & 5.1948 & 117 & 102 & -6.0241 & 12.8205 & 100 & 91 & 0.5025 & 9 \\
\hline IR 47-701-6-3-1 & $\mathrm{X}$ & IRAT 239 & 22.31 & 21.32 & 4.4375 & 4.4375 & 110 & 100 & 8.3744 & 9.0909 & 91 & 87 & 1.6760 & 4.3956 \\
\hline F-LSD $(\mathrm{p}=0.05)$ & & & 2.909 & 2.55 & - & - & 17.550 & 18.233 & - & - & 13.043 & 15.335 & & \\
\hline
\end{tabular}


Onyia $^{1}$, V.N., Obi, ${ }^{1}$ I.U and Anyanwu ${ }^{2}$ C. P

Table 3 (cont). Mean values of the agronomic characters of the parents, $F_{1}$ 's, $F_{2}$ 's , mid parent heterosis (\% het) for $F_{1}$ hybrids and Inbreeding depression ( $\%$ ID for $F_{2}$ populations) in the rice breeding lines.

\begin{tabular}{|c|c|c|c|c|c|c|c|c|c|c|c|c|c|c|}
\hline & & & \multicolumn{3}{|c|}{ Number of days to maturity } & \multicolumn{3}{|c|}{$\underline{\text { 1000-grain weight (g) }}$} & \multicolumn{4}{|c|}{ Grain yield (tons/ha) } & & \\
\hline Cro & $\mathrm{s}$ & Ses & $\mathrm{F}_{1}$ & 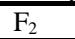 & $\% \mathrm{Het}$ & $\% \mathrm{ID}$ & 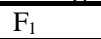 & $\mathrm{F}_{2}$ & $\% \mathrm{Het}$ & \%\%ID & $\overline{F_{1}}$ & $\mathrm{~F}_{2}$ & $\% \mathrm{Het}$ & $\% \mathrm{ID}$ \\
\hline WAB 450-1-B-163-41 & $\mathrm{X}$ & WAB35-2-FX & 98 & 98 & -4.854 & 0 & 34.92 & 32.15 & -0.14 & 6.405 & 4.96 & 4.72 & -4.798 & -11.34 \\
\hline WAB 450-1-B-163-41 & $\mathrm{X}$ & WAB56-144-FX & 101 & 102 & -2.415 & -0.901 & 34.02 & 33.00 & 0.502 & 2.998 & 4.2 & 3.95 & -11.0 & 5.952 \\
\hline WAB 450-1-B-163-41 & $\mathrm{X}$ & WAB 56-100 & 100 & 100 & -0.990 & 0 & 34.72 & 30.35 & 0.842 & 7.932 & 4.22 & 4.05 & -28.10 & 4.838 \\
\hline WAB35-2-FX & $\mathrm{X}$ & WAB56-144-FX & 104 & 104 & -1.421 & 0 & 35.01 & 35.02 & 1.625 & -0.02 & 4.2 & 4.29 & 3.448 & -2.142 \\
\hline WAB35-2-FX & $\mathrm{X}$ & WAB 56-100 & 103 & 102 & 0.9709 & 0.9709 & 34.71 & 32.92 & 4.453 & 5.157 & 4.1 & 3.85 & 0 & 6.098 \\
\hline WAB56-144-FX & $\mathrm{X}$ & WAB 56-100 & 105 & 103 & 2.4390 & 1.9048 & 32.81 & 32.60 & 2.371 & 0.640 & 3.72 & 3.60 & 3.047 & 3.2258 \\
\hline WAB 450-1-B-163-41 & $\mathrm{X}$ & IRAT 317 & 112 & 111 & 1.3575 & 0.8929 & 35.33 & 30.21 & 22.80 & 14.49 & 3.84 & 3.68 & -11.52 & 4.1667 \\
\hline WAB 450-1-B-163-41 & $\mathrm{X}$ & WAB35-1-FX & 104 & 105 & -3.255 & -0.901 & 3.01 & 35.26 & -2.35 & 2.082 & 4.15 & 4.112 & -11.51 & 0.722 \\
\hline WAB $450-1-B-163-41$ & $\mathrm{X}$ & IR 47-701-6-3-1 & 108 & 108 & 0 & -0.901 & 32.31 & 29.66 & 12.77 & 2.082 & 3.62 & 3.45 & -11.49 & 0.722 \\
\hline WAB $450-1-B-163-41$ & $\mathrm{X}$ & IRAT 239 & 108 & 107 & 1.8868 & 0 & 35.21 & 34.16 & 18.03 & 8.201 & 3.80 & 3.60 & -12.84 & 4.698 \\
\hline WAB35-2-FX & $\mathrm{X}$ & IRAT 239 & 101 & 104 & -6.481 & 0.9259 & 34.61 & 33.00 & 13.73 & 2.982 & 3.45 & 4.11 & -6.756 & 5.263 \\
\hline WAB56-144-FX & $\mathrm{X}$ & IRAT 239 & 101 & 104 & -6.912 & -2.970 & 33.05 & 33.00 & 12.99 & 4.651 & 3.00 & 2.89 & -6.542 & -19.1 \\
\hline IRAT 317 & $\mathrm{X}$ & WAB35-2-FX & 115 & 113 & 2.222 & 1.7391 & 27.11 & 27.00 & -7.694 & 0.4058 & 3.82 & 3.80 & 3.8043 & 0.5236 \\
\hline IRAT 317 & $\mathrm{X}$ & WAB56-144-FX & 116 & 114 & 2.6549 & 1.7241 & 28.01 & 26.73 & -0.603 & 4.5698 & 3.40 & 3.32 & 6.5831 & 2.3529 \\
\hline IRAT 317 & $\mathrm{X}$ & WAB 56-100 & 119 & 114 & 0.9925 & 4.2017 & 27.10 & 26.23 & 0.4820 & 3.2103 & 3.58 & 3.51 & 10.8359 & 1.9553 \\
\hline WAB35-1-FX & $\mathrm{X}$ & WAB35-2-FX & 111 & 110 & 1.3699 & -0.9009 & 38.60 & 36.32 & 2.9883 & 5.9067 & 4.25 & 4.16 & 1.1905 & 2.1176 \\
\hline WAB35-1-FX & $\mathrm{X}$ & WAB56-144-FX & 101 & 103 & -8.1818 & -1.9802 & 37.21 & 35.68 & 2.5069 & 4.1112 & 9.225 & 9.11 & -9.9730 & -2.0958 \\
\hline WAB35-1-FX & $\mathrm{X}$ & WAB 56-100 & 113 & 109 & 6.1033 & 3.5398 & 40 & 39.69 & 14.606 & 0.775 & 3.94 & 3.61 & 5.0667 & 8.3756 \\
\hline IR 47-701-6-3-1 & $\mathrm{X}$ & WAB35-2-FX & 110 & 110 & 0 & 0 & 24.10 & 23.10 & -17.60 & 4.1493 & 3.30 & 3.20 & -3.7901 & 3.0303 \\
\hline IR 47-701-6-3-1 & $\mathrm{X}$ & WAB56-144-FX & 112 & 111 & -4.7619 & 17.1429 & 27.11 & 26.28 & -7.692 & -16.666 & 2.80 & 2.32 & -4.7619 & 17.1429 \\
\hline IR 47-701-6-3-1 & $\mathrm{X}$ & IRAT 239 & 110 & 108 & 1.3423 & 0.6623 & 26.61 & 25.62 & 7.6923 & 0 & 3.02 & 3.00 & 1.3423 & 0.6623 \\
\hline IRAT 239 & $\mathrm{X}$ & WAB 56-100 & 110 & 107 & 4.5383 & -5.5625 & 25.81 & 25.21 & 20 & 0 & 3.20 & 3.25 & 0.6623 & -5.5623 \\
\hline IRAT 317 & $\mathrm{X}$ & WAB35-1-FX & 108 & 98 & 2.5641 & 1.6667 & 28.10 & 26.20 & -9.9936 & 7.5630 & 2.96 & 2.81 & -11.111 & 2.8125 \\
\hline IRAT 317 & $\mathrm{X}$ & IR 47-701-6-3-1 & 98 & 81 & 2.128 & 1.6667 & 23.80 & 22.00 & 3.5233 & 6.7616 & 3.20 & 3.11 & 25 & 5.0676 \\
\hline IRAT 317 & $\mathrm{X}$ & IRAT 239 & 108 & 101 & 2.164 & 1.6949 & 24.01 & 22.45 & -0.6620 & 6.4973 & 2.97 & 2.95 & 4.9470 & 0.6734 \\
\hline WAB35-1-FX & $\mathrm{X}$ & IR 47-701-6-3-1 & 114 & 100 & -3.9301 & -1.8182 & 39.41 & 38.00 & 26.7202 & 3.5778 & 2.92 & 2.90 & -5.1948 & 0.6849 \\
\hline WAB35-1-FX & $\mathrm{X}$ & IRAT 239 & 117 & 102 & 2.2222 & 1.7391 & 38.01 & 35.67 & 17.7509 & 29.2135 & 3.10 & 3.05 & -7.4627 & 1.6129 \\
\hline IR 47-701-6-3-1 & $\mathrm{X}$ & IRAT 239 & 110 & 100 & 0.8850 & 0.8772 & 23.91 & 21.92 & -0.5821 & 12.5052 & 2.96 & 2.82 & 14.728 & 4.7297 \\
\hline F-LSD $(p=0.05)$ & & & 0.685 & 0.79 & - & - & 1.8604 & 1.4566 & - & - & 0.4865 & 0.5342 & - & - \\
\hline
\end{tabular}


This indicates that these crosses could be good materials for developing high yielding hybrids because panicle number, total dry mater and spikelet number/grain number per panicle reportedly contributes greatly to high grain yield production (Dwivedi, et al., 1998). Earlier, negative heterosis for panicle number had been reported by Virmani et al., $(1981,1982)$ and Jennings (1967). The use of the number of panicles alone is not enough in determining yield in Oryza spp. Gravais and McNew (1993) have earlier suggested that the selection for increased yield via selection for either panicle weight or panicle number alone would be in effective. Therefore, selection for both increased panicle weight and panicle number to increase yield was estimated to be $91 \%$ as effective as selecting for yield directly (Surek and Beser, 2005).

Hybrid vigor for panicle length was observed in some of the crosses such as WAB 35-1-FX X WAB 56-100 and WAB 35-1-FX X IR 47-701-6-3-1. The result also show that some of the crosses that had high tillering ability also have an appreciable increase in terms of grain yield. The hybrids WAB 35-1-FX X WAB 35-2-FX and IRAT $317 \mathrm{X}$ WAB 35-1-FX had the highest number of tillers/plant of 15 each in the $F_{1}$ progeny. The hybrid IRAT $317 \mathrm{X}$ WAB35-1-FX also had the highest number of tillers/plant of 14 in the $F_{2}$. The result presented in Table 3 show that the hybrids WAB 144-FX X WAB 56-100 and WAB 56-144-FX X IRAT 239 had the highest heterotic values of $26.31 \%$ each. This is in line with the report of Basavavaja et al., (1998) that productive tillers/plant can have a high positive effect contribution towards grain yield per plant. The result also agrees with the findings of Ibrahim et al. (1990) that productive tillers was one of the most reliable character in selecting genotypes of rice.

The results obtained, suggests that heterosis in yield were due to yield components like tiller number, panicle length, spikelet number and 1000-grain weight. Grafius (1959) had earlier suggested that there is no separate gene system for yield per second and that the grain yield is an end product of the multiplication interaction between the yield components. This was, confirmed by the present research where more showed hybrid vigor for yield alone. Hybrid vigor for yield is the result of interaction of simultaneous increase in the expression of yield components.

Inbreeding depression was not found to be significant in most of the studied characters. Positive (ID) in $\mathrm{F}_{2}$ generation was observed in the characters of tiller number per plant, panicle length, spikelet fertility, 1000 grain weight and grain yield resulting is it hybrid vigour hybrid.
However some of the hybrids such as WAB 35 1-2FX x WAB 56-144-FX and R 47-701-6-3-1$x$ IRAT 239 exhibited a low level of inbreeding depression for yield characters such as panicle number and 1000 grain weight showing their high level of stability as $F_{1}$ variety. Morrover, hybrid break down in self pollinated plant species such as rice has been observed by many researchers (Li et al;; 1997a ; b and 1997b).

\section{CONCLUSION}

The results of the investigation showed that $F_{1}$ rice hybrids are useful not only for their high grain yield per cropping season but also the possibility of obtaining more heterotic hybrids in specific cross combinations with them. The findings that most of the $F_{1}$ hybrids were superior to their $F_{2}$ populations and $F_{2}$ populations showed considerable inbreeding depression in majority of the cases, thus, the possibility of getting $F_{2}$ seed with a performance of anyway near, $F_{1}$ seed is not feasible.

It can be concluded from this study that with appropriate choice of parental lines, it is possible to develop $F_{1}$ rice hybrid possessing distinct yield superiority over the best-inbred lines.

\section{REFERENCES}

Ahmad, M.I. (1996) Hybrid rice production technology DRR, Hyderabad.

Anandakumar, C.R. and Sree Rangasamy, S.R. (1986). Heterosis and Inbreeding depression in rice. Oryza 23: 96-101.

Basavaraja, P., Rudraradlya, M and Kulkarni, R.S. (1998) Genetic variability, correlation and path analysis of yield components in two $\mathrm{F}_{4}$ population of fine grain rice. Mysore Journal of Agricultural Sciences (1997). 31 (1) 16.

Birchler, J.A., Donald, L.A. and Riddle, N. (2003). In search of Molecular basis of heterosis. Plant Cell 15: 2236-2239

Dwivedi, D.K., Pandey, M.P., Pandey, S.K. and Rongbai, .I. (1998). Heterosis over environment in crosses involving indica and tropical Japonica rice cultures. IRRI Notes. Vol 23 number $2 \mathrm{p}$

Falconer, D.S. and F.C. Mackey (1996) Introduction to quantitative genetics. Fourth edition. Longman. New York.

Gravais, K.A. and McNew. R.M. (1993). Genetic relationships and selection for rice yield and yield components. Crop Sci. 33: 259-262.

Ibrahim, S.M. Ramalingam, A and Subramanian, A. (1990) Path analysis 
of rice grain yield under rained lowland conditions IRRI 15(1): PP11

Jennings, P.R. (1967). Rice Heterosis at different growth stages in a tropical environment. Int. Rice. Comm. Newsl. 16(2): $24-26$

Jones, J.W. (1926) Hybrid vigour in rice. Journal of the American Society of Agronomy 18: 423-428.

Khaleque, M.A., O.I., Jorller and A.M., Eunus. (1977). Heterosis and Combining ability in diallel cross of rice (Oryza saliva L.) Bangla, J. Agric. Acc. 4: 137-145.

Li, Z.K., Pinson, S.R.M., Paterson, A.H., Paric, N.D. and Starsel, J.W. (1997a) Epistassi For three grain yield component in rice (Oryza sativa. L) Genetics, 145: 453-465.

Li, Z.K., Pinson, S.R.M., Paterson, A.H., Paric, N.D. and Starsel, J.W. (1997b) Genetics of hybrid sterility and hybrid breakdown in an inter-sub specific rice (Oryza sativa. L) Population Genetics, 145: 1139-1148.

Mohammad, K.C. and Mohanty, M.K. (1992). Inheritance of some qualitative characters including heterosis in rice by combining ability analysis inheritance of quantitative traits in rice genetic. IRRI Notes Vol 11: p 14.

Nuruzzaman, M. Alam, M.F., Ahwo., M.G. Shohael, M.A., Biswas, M.K., Amin, M.R. and Hossain, M.M. (2002). Studies on parental variability and heterosis in rice (Oryza sativa L.) Pak. J. Biol. Sci. (10): 1006-1009.
Obi, I.U. (2003). Statistical Methods of detecting differences between treatment means SNAP Press Ltd Enugu. Nigeria PP 45.

Shull, G.H. (1908). The combination of a field of maize. Res. of American Breed Assoc. 4: 296-301.

Singh, S.P., Singh, .R.R., Singh R.P, and Singh R.V. (1980) Heterosis in rice. Oryza 17 109-113.

Sitaramakah, C.H., Rani, V.D. and Roddi, N.S. (1998) Standard heterosis of rice hybrid for yield and yield components IRRI notes Vol 23 p 15.

Steel, C.D. and Torres, J.K. (1980). Principles and procedures of statistics. A biometrical Approach, second edition Mc Graw. Hill books Co. Inc. New York. 48/pp.

Surek H and Beser N. (2005). Selection for grain yield and its component in early generation in rice. (Oryza sative L.) Trakya Univ. J. Sci 6(1) 51-58.

Virmani, S.S., Aquino R.C. and Khush G.S. (1982). Heterosis breeding in rice. Oryza sativa L. Theo. Appl. Genet. 63: 373-380.

Virmani, S.S., Chaamphony, R.C. and Khush, G.S. (1981) Current outlook on hybrid rice. Oryza 18; 67-84.

Vive Kananden R and Giridheram, S. (1995) Heterosis for Kernel Characters in rice IRRI Notes 20. P1

Wynne, J.C., Emery, D.A and Rice, P.N. (1979). Combining ability estimates in Arachis hypogaea $\mathrm{L}$. II field performance of $\mathrm{F}_{1}$ hybrids. Crop Science 10: 713-715. 